\title{
Integrated Polysilicon and DRIE Bulk Silicon Micromachining for an Electrostatic Torsional Actuator
}

\author{
Jer-Liang Andrew Yeh, Student Member, IEEE, Hongrui Jiang, Student Member, IEEE, \\ and Norman C. Tien, Member, IEEE
}

\begin{abstract}
This paper presents a fabrication process that integrates polysilicon surface micromachining and deep reactive ion etching (DRIE) bulk silicon micromachining. The process takes advantage of the design flexibility of polysilicon surface micromachining and the deep silicon structures possible with DRIE. As a demonstration, a torsional actuator driven by a combdrive moving in the out-of-plane direction, consisting of polysilicon fingers and bulk silicon fingers, has been fabricated. The integrated process allows the combdrive to be integrated with any structure made by polysilicon surface micromachining. [433]
\end{abstract}

Index Terms - Asymmetric combdrive, electrostatic torsional actuator, integrated polysilicon and deep reactive ion etching (DRIE) bulk silicon micromachining, levitation, parallel-plate actuator.

\section{INTRODUCTION}

$\mathbf{S}$ ILICON micromachining has been utilized since the early 1960 's. At its early stage, anisotropic single-crystal silicon etching technology was employed in the majority of the research efforts. In the early 1980's, surface micromachining using sacrificial etching gave rise to new types of microsensors and microactuators. More recently, deep reactive ion etching (DRIE) technology has given tremendous impetus to highaspect-ratio dry etching of single-crystal silicon.

DRIE bulk silicon micromachining is a fabrication technique that allows one to produce deep structures in a silicon substrate or even movable structures by the use of undercuts. Many fabrication methods have been used to make microstructures out of single-crystal silicon, including single-crystal silicon reactive ion etch and metallization (SCREAM) process [1], deep-etch shallow-diffusion process [2], and silicon-oninsulator (SOI) process. These techniques have several common advantages such as excellent mechanical properties and high-aspect-ratio structures. However, there exist geometric constraints to the types of structures that can be fabricated; some examples are limitations on the maximum width of released beams, difficulty with the integration of membranes parallel to the substrate, and inability to create multiple-level structures with various features (e.g., hinges).

The purpose of polysilicon surface micromachining is to fabricate micromechanical structures from deposited thin films.

Manuscript received March 22, 1999; revised August 2, 1999. Subject Editor, E. Obermeier.

The authors are with Cornell University, Ithaca, NY 14853 USA

Publisher Item Identifier S 1057-7157(99)09611-0.
Polysilicon structures anchored to the silicon ground plane are free to move after the underlying sacrificial material is removed. Multiple layers of structural polysilicon and sacrificial layers can be sequentially deposited and patterned in order to realize complex and multilayered structures [3]. Three-dimensional polysilicon structures can be built by folding out the surface structures. Various types of actuators such as the electrostatic interdigitated combdrive [4], the scratch drive actuator [5], and the thermal actuator [6] have been developed to move these microstructures. However, the thinness of the deposited polysilicon film can limit aspects of the device performance such as capacitive sensing signal, electrostatic force, and resonant frequency. In addition, the use of polysilicon brings up some material and fabrication issues that do not appear in bulk micromachining processes such as residual stress, stress gradient through the film, variation of Young's modulus, topography with multiple-layer structures, and in-use/release stiction.

Both DRIE bulk silicon micromachining and polysilicon surface micromachining have merits and demerits when used alone. However, many limitations of one method can be overcome by the other. Integrated polysilicon and DRIE bulk silicon micromachining not only retains the advantages of both methods but also expands the range of structure designs. In addition to the structures that can be made by the individual methods, combinations of surface and bulk methods can be applied to build actuators such as combdrives with fingers asymmetric in height and thickness, parallel-plate actuators, anisotropic springs, and mixtures of beams and membranes [7]. Furthermore, the resonant frequencies of these structures can be designed to be far apart from each other by having them made using either polysilicon surface micromachining or DRIE bulk silicon micromachining. The separation of resonant frequencies benefits structural stability and reduces coupling between structures.

\section{Device Demonstration}

To demonstrate the feasibility of integrated polysilicon and DRIE bulk silicon micromachining, we have fabricated an electrostatic torsional actuator driven by an asymmetric combdrive, shown in Fig. 1, which generates a torque on a polysilicon membrane. Unlike a conventional combdrive where the fingers of both combs are of the same thickness and in the same plane, the distinction of the combdrive we 


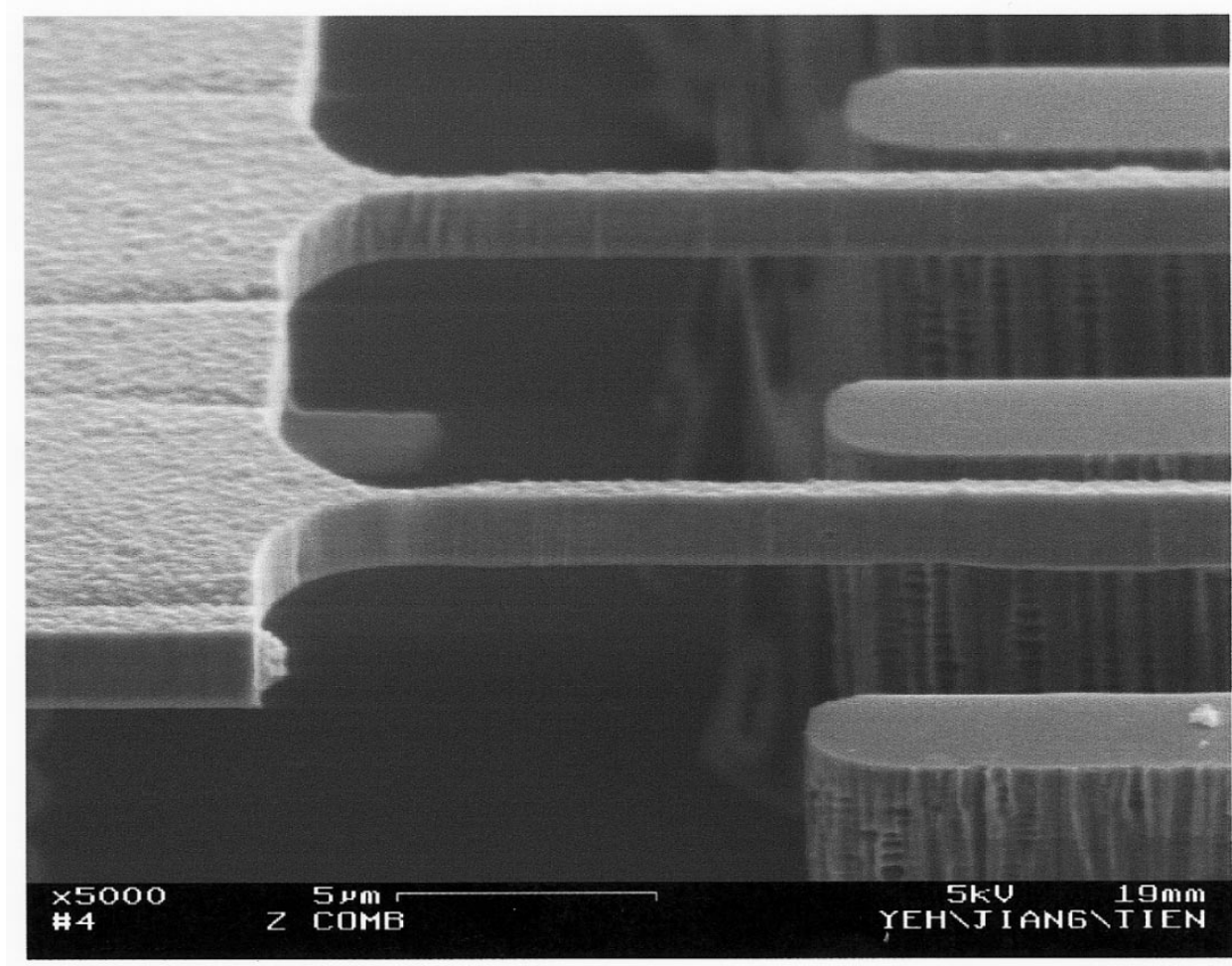

Fig. 1. Closeup SEM of a fabricated asymmetric combdrive formed by (upper) polysilicon fingers and (lower) bulk silicon fingers.

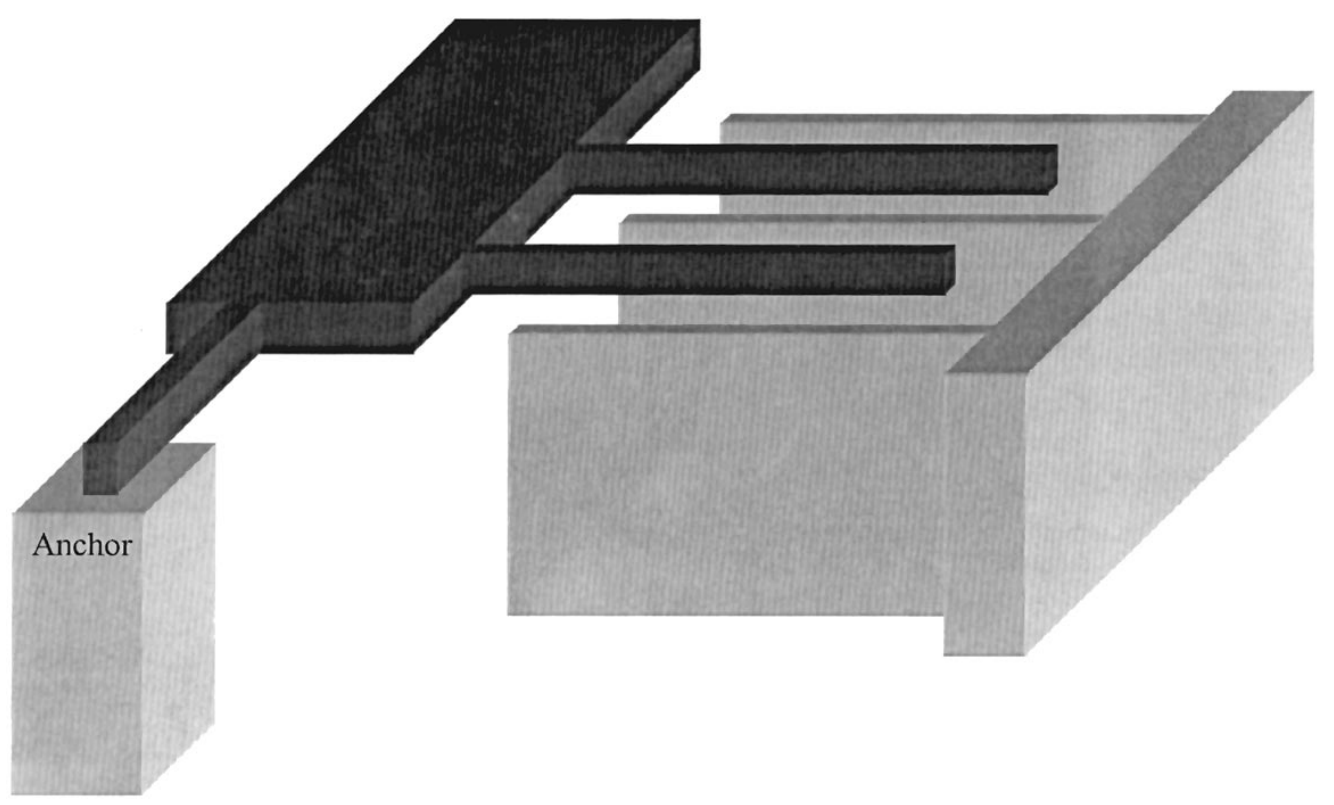

Fig. 2. Schematic of a device actuated by asymmetric combdrives.

present is that the fingers on one comb are thinner and higher than those on the other comb. Actuation is achieved when the thin polysilicon fingers are pulled downward toward the substrate with respect to the fixed thick bulk silicon fingers under a voltage applied between the combs (see Fig. 2). The pulldown of the movable comb is the result of fringing electric field, shown in Fig. 3, on the polysilicon fingers, which creates torsional motion. The asymmetry in height and thickness of comb fingers causes a net fringing capacitance that points down into the substrate. Note that the actuator's differential capacitance varies with the position of the movable polysilicon fingers. Hence, this combdrive cannot generate a constant output torque due to its position-dependent differential capacitance.

Present approaches for producing torsional motion by electrostatic actuators include parallel-plate actuators [8]-[13], in-plane interdigitated combdrives utilizing the out-of-plane fringing force [14], [15], and in-plane interdigitated comb- 


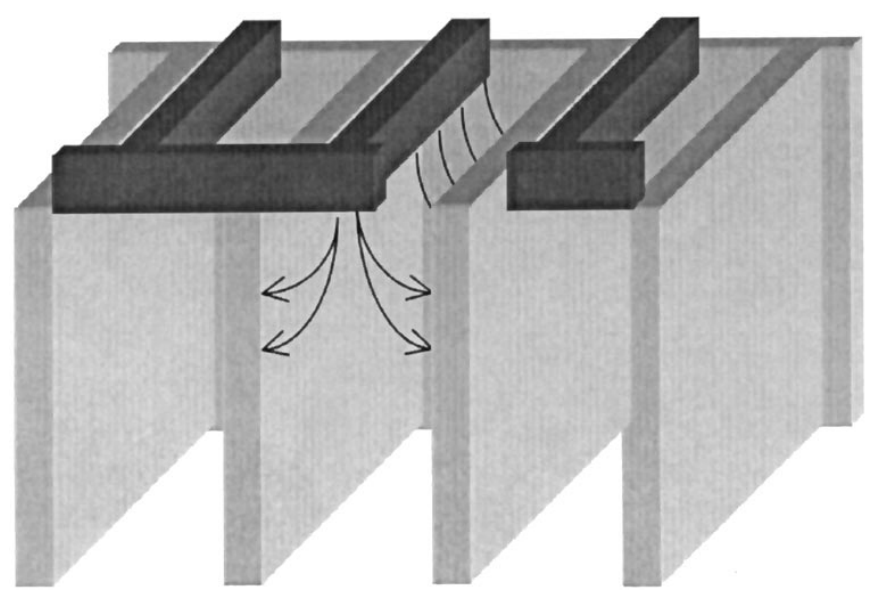

Fig. 3. Schematic of the electric field lines in an asymmetric combdrive.

drives used to swing structures such as a plate connected to it by a hinge. An example is the folded-up scanning mirrors driven by combdrives [16], [17]. In those structures, dynamic problems such as coupling, backlash, and low resonant frequency can be encountered. Coupling between torsional and translational motions results in the instability of the device. Backlash occurs at the moment when the direction of motion is changing because of the clearance between the pin and the staple. In devices where torsional motion can be generated by in-plane interdigitated combdrives, the maximum angle of rotation is limited.

Typically, large rotational motion is achieved using a parallel-plate actuator. Asymmetric combdrives fabricated by integrated polysilicon and DRIE bulk silicon micromachining are an alternative that offers better dynamic performance for a large rotational motion. The static and dynamic performance of a parallel-plate actuator and our combdrive are discussed in Section IV. Given constraints on rotation angle and resonant frequency, it is found that a membrane will twist at a lower operating voltage with this asymmetric combdrive than with a parallel-plate actuator [18].

With our new technology, we have fabricated a device, shown in Fig. 4, that uses these combdrives to actuate a polysilicon membrane in torsional motion. The membrane is 200 by $200 \mu \mathrm{m}^{2}$, and its attached springs are $200 \mu \mathrm{m}$ long and $2 \mu \mathrm{m}$ wide from the top view. Both the membrane and the springs are fabricated from polysilicon of thickness $1.5 \mu \mathrm{m}$. In addition, we have created another device (see Fig. 5) that has additional silicon parallel-plate electrodes of width $85 \mu \mathrm{m}$, which are $20 \mu \mathrm{m}$ below the membrane. The membrane and its underlying electrodes can be used either as a parallel-plate actuator or as a capacitive sensor. Both devices are fabricated on a silicon-on-insulator (SOI) wafer. The bulk fingers and underlying electrodes are fabricated from the silicon layer of the SOI wafer. On top of the SOI wafer, surface micromachining is used to form the polysilicon structures.

The polysilicon membrane, shown in Fig. 4, is suspended by a pair of rectangular bars and is actuated by our asymmetric combdrive. The membrane is intended as a scanning micromirror. The scanning micromirror has many applications such as laser beam positioning, laser beam steering, and fiber-optic switching [9], [10], [16], [17], [19]. Two sets of combdrives are placed at both ends of the micromirror parallel to the substrate. The torsional springs, micromirror, and movable fingers of the combdrive are made of polysilicon. The top silicon layer of the SOI wafer is used to form the fixed fingers of the combdrive. The induced fringing electric field lines on one side of the combdrive generate a downward force and a torque on the polysilicon suspension springs. The force lowers the position of the micromirror with respect to the anchored end of the springs, while the torque causes an into-plane rotational motion of the micromirror, allowing it to twist around the polysilicon torsional springs.

A device consisting of the previous structure and additional parallel plates is shown in Fig. 5. The parallel plates can be used for precise sensing and control of angular motion and position. This device can be driven by both actuators or only one while the other actuator is used as a sensor to form an internal feedback loop. Potentially, either the asymmetric combdrive or the parallel-plate actuator can be used to provide the rotational motion of the micromirror, while the other can be employed as a differential capacitive sensor to detect angular changes. This feedback system can be applied to control either angular position or angular velocity of a micromirror.

\section{FABRICATION}

The fabrication processes for the devices (see Figs. 4 and 5) will be described in this section. The only difference between these two processes is that the process for the device with underlying electrodes (Fig. 5) requires one more etch step to form an oxide block with two different depths. In general, the mechanical structures were built not only from thin films deposited on top of the silicon surface but also in the top silicon layer of a SOI wafer. The key to the processes is the formation of a sacrificial oxide block prior to the polysilicon surface micromachining [20]. The oxide block formation starts with the etching of closely spaced trenches in the silicon layer of the SOI wafer. Thermal oxidation transforms the remaining silicon into silicon dioxide. Finally, low-pressure chemical vapor deposition (LPCVD) oxide is deposited to refill the trench openings, and after planarization the oxide block is created. Details of the process are described below.

This approach to the formation of the oxide block has several advantages over deposited oxide refilling of wide and deep trenches. First, only a few-micrometers-thick oxide deposition is required for the trench opening refill no matter what the depth is because of the closely spaced thermal oxide posts. Furthermore, a thinner film deposition results in a smoother topography on the wafer, making planarization easier. In addition to the process advantages, a thin oxide deposition also reduces the induced moment acting on the substrate that is caused by the stress distribution through the film, which depends on the film thickness. A large bending moment would severely bend the wafer. The residual stress and built-in stress gradient of a thick deposited oxide may also crack the oxide film. In general, the problems caused by stress distribution within the deposited thin films are highly 


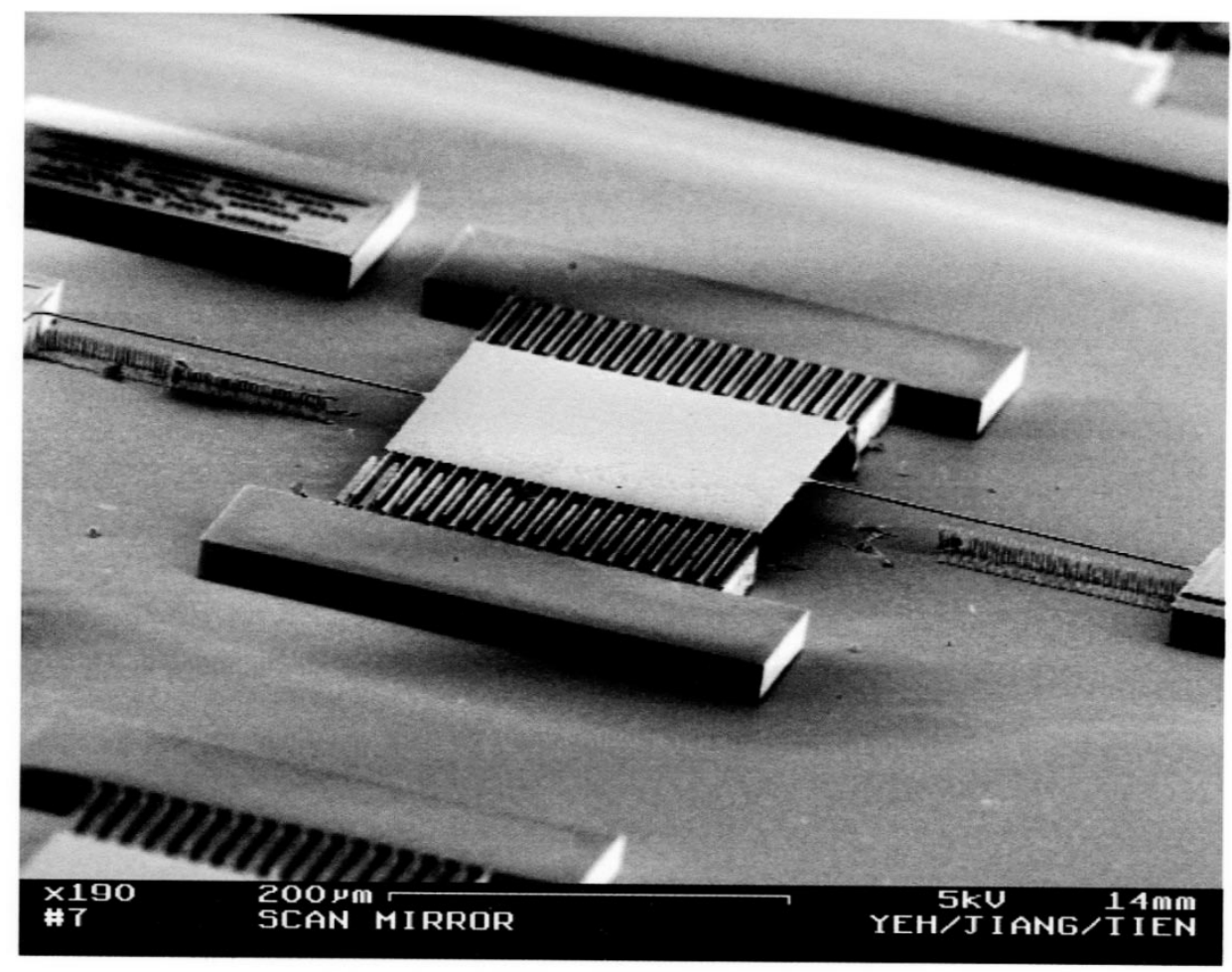

Fig. 4. SEM of a fabricated asymmetric-combdrive actuated membrane.

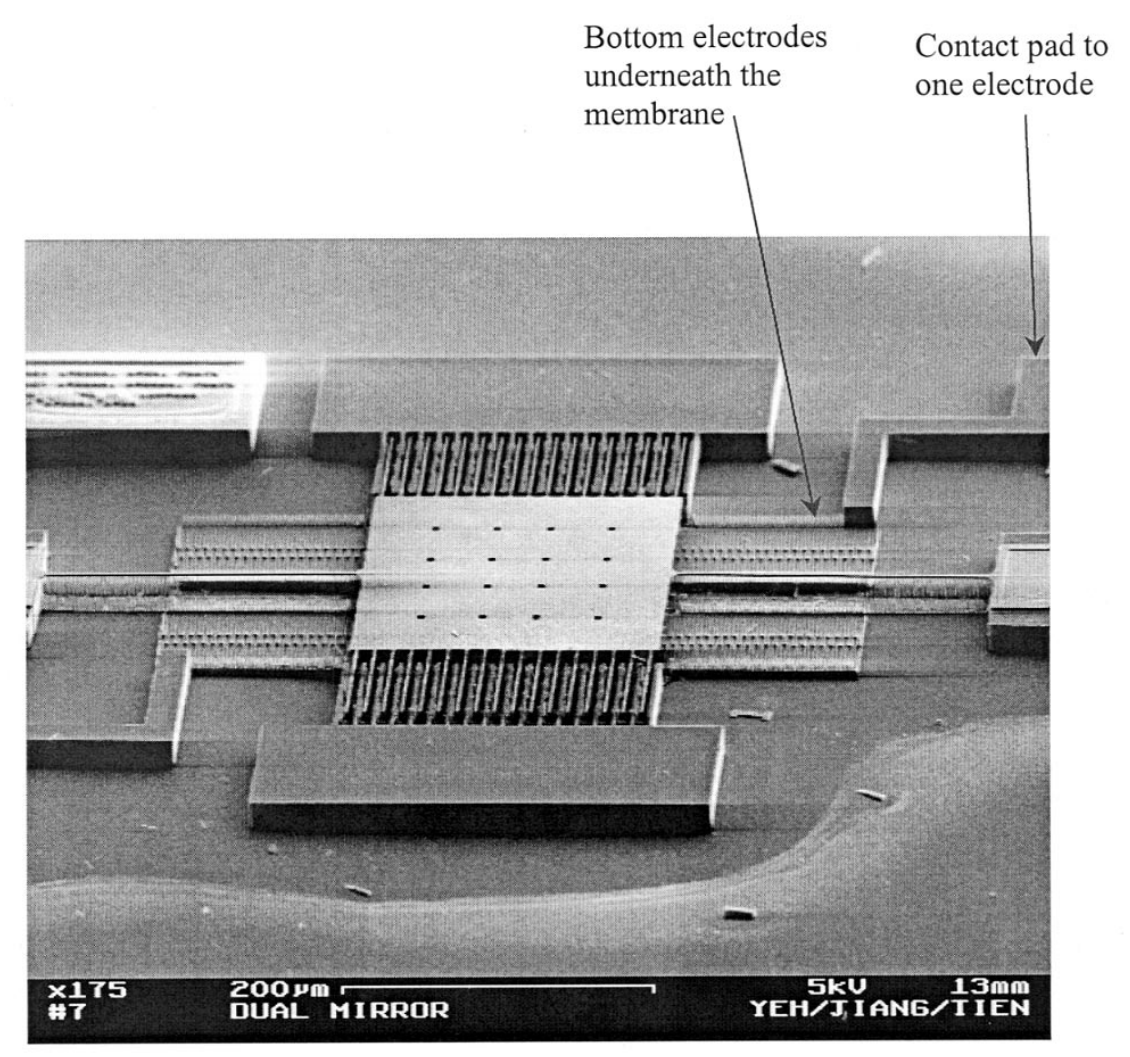

Fig. 5. SEM of a fabricated dually actuated membrane. The etch holes are used to speed up the wet etch release of the structures.

dependent on the film thickness and greatly reduced with our technique.

\section{A. A Membrane Actuated by Asymmetric Combdrives}

The process sequence used to fabricate the membrane and asymmetric combdrives (see Fig. 4) is depicted schematically 


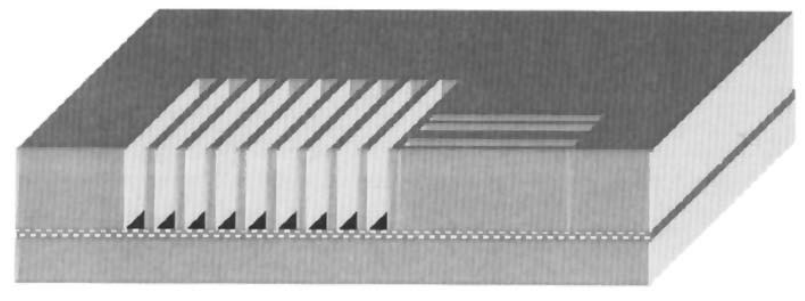

(a)

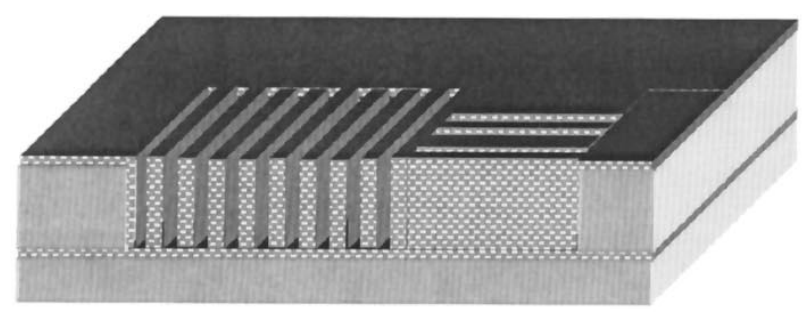

(b)

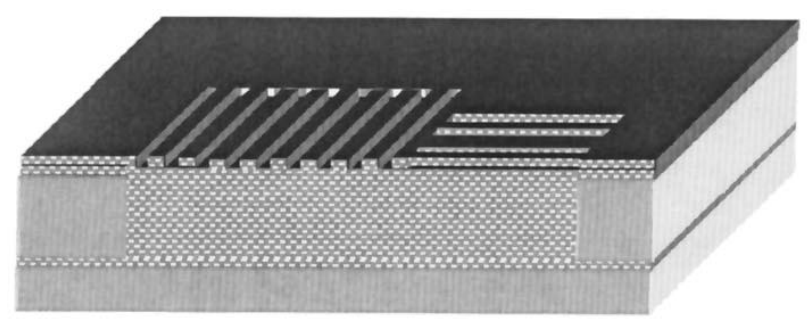

(c)

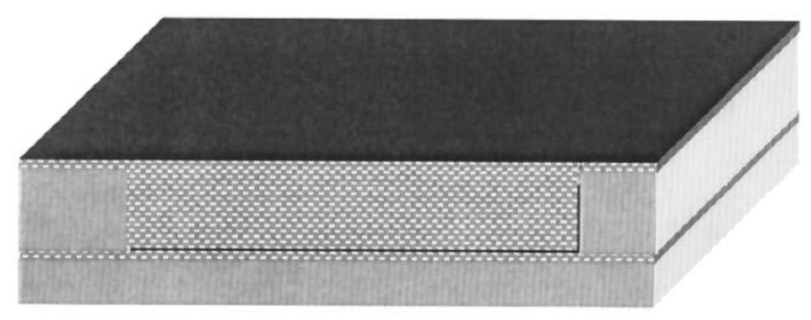

(d)

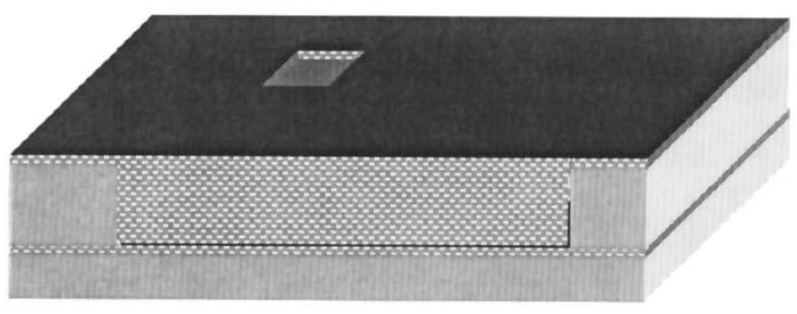

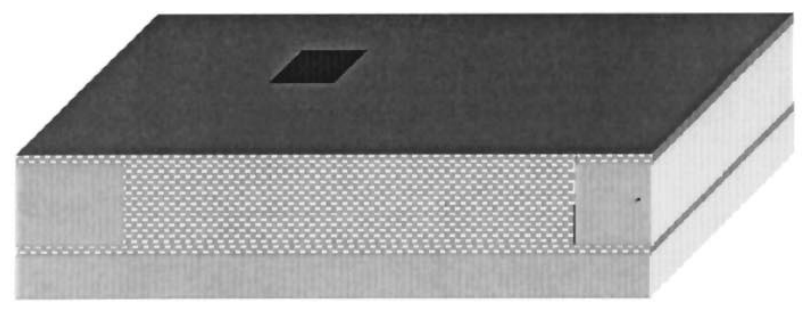

(f)

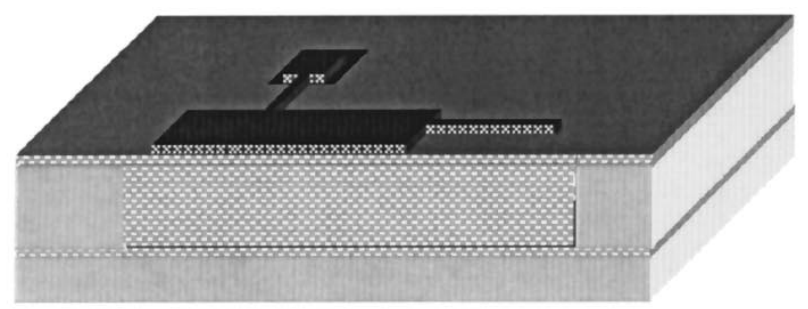

(g)

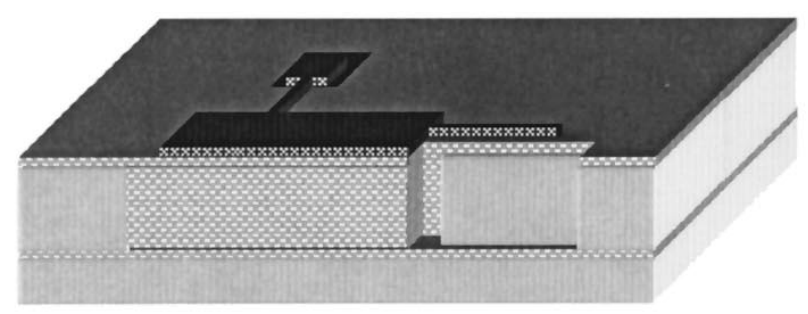

(h)

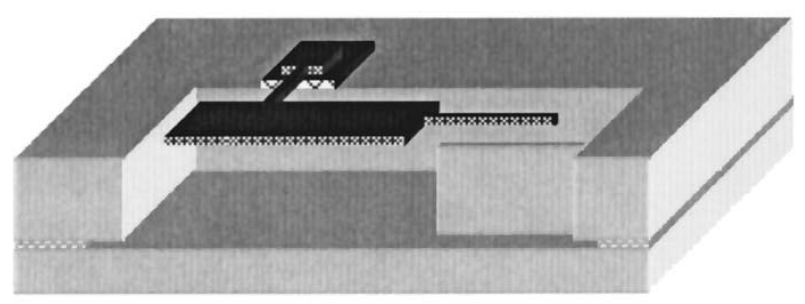

(i)

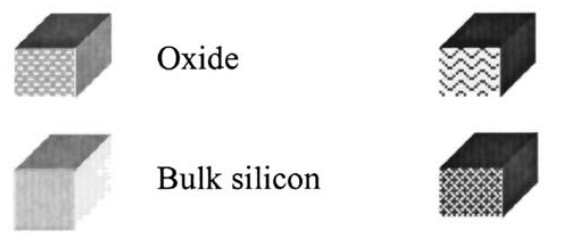

Nitride

Polysilicon

(e)

Fig. 6. Fabrication sequence for the asymmetric-combdrive actuated membrane. (a) Pattern and etch beam structures of an oxide block. (b) Thermal oxidation at the temperature of $1050{ }^{\circ} \mathrm{C}$. (c) Refill trenches with LPCVD oxide. (d) Chemical mechanical polishing. (e) Pattern and nitride anchors through the sacrificial oxide. (f) Deposit 3000 A low- $\sigma$ nitride and define and isolation regime. (g) Deposit 1.5- $\mu$ m-thick polysilicon and define structures such as a membrane, hinges. (h) Define combdrives and etch through the layers: polysilicon, oxide, and the top silicon layer of the SOI wafer. (i) HF solution is used to release the membrane and combdrives.

in Fig. 6. We began with an SOI wafer that had a $20-\mu$ mthick silicon layer on top of a $1-\mu \mathrm{m}$-thick buried oxide. First, trenches that were etched in the top silicon layer with a DRIE etcher (PlasmaTherm SLR770) using photoresist as the mask were 2-3 $\mu \mathrm{ms}$ wide, $1 \mu \mathrm{m}$ apart, and $20 \mu \mathrm{m}$ deep [see Fig. 6(a)]. The beams were completely thermally oxidized at a temperature of $1050{ }^{\circ} \mathrm{C}$; the thermal oxidation transformed silicon to silicon dioxide resulting in a slightly larger oxide block than the dimension in the design [see Fig. 6(b)]. Following the oxidation step, the open trenches between the fully oxidized beams were filled with conformal LPCVD oxide $(4 \mathrm{~nm} / \mathrm{min}$ at $900{ }^{\circ} \mathrm{C}$ ), resulting in ripples on the oxide surface [see 
Fig. 6(c)]. These ripples were then planarized with chemicalmechanical polishing (CMP) [see Fig. 6(d)]. The combination of thermally grown oxide and deposited oxide served as an oxide block for the subsequent surface micromachining. The oxide block formation transformed the unwanted single-crystal silicon to silicon dioxide, which could be removed in the final step of structure release. The formation sequence of one layer of the oxide block is illustrated in Fig. 6(a)-(d).

Conventional polysilicon surface micromachining was subsequently performed on top of the wafer. The oxide block helped maintain a flat surface across the substrate for the following thin film processing. A $1.1-\mu \mathrm{m}$-thick LPCVD oxide layer was deposited to form the first sacrificial layer. Windows were dry etched in the LPCVD oxide with a flourine-based etcher so that afterwards a low-stress nitride and polysilicon deposition could be anchored onto the substrate [see Fig. 6(e)]. A 250-nm low-stress nitride layer was used as an isolation layer between the polysilicon and the top silicon layer of the SOI wafer. This layer was then photolithographically patterned and etched [see Fig. 6(f)].

The structural layer is $1.5-\mu \mathrm{m}$-thick LPCVD in situ borondoped polysilicon deposited at a temperature of $620^{\circ} \mathrm{C}$ [see Fig. $6(\mathrm{~g})]$. The polysilicon layer was used to form the structures such as membrane and springs and to provide the material for the movable fingers of the asymmetric combdrives, which were etched in the final deep etch. This layer was anchored onto a stationary bulk silicon piece. A 450-nm overlay oxide was deposited and thermally annealed at the temperature of $1000{ }^{\circ} \mathrm{C}$ for $1 \mathrm{~h}$. The deposited oxide was patterned and used as a hard mask for etching the polysilicon underneath with a chlorine-based etcher. Another 350-nm oxide was subsequently deposited to serve as a hard mask for the singlecrystal silicon structure etch.

Following the thin-film processing, a final mask was used to pattern the top silicon layer on the SOI wafer and the thinfilm layers on top of it [see Fig. 6(h)]. For example, in the formation of the asymmetric combdrives, both the bulk silicon fingers and the polysilicon fingers were defined in the same lithographic step to prevent lateral misalignment between the two combs, which would severely degrade its performance. With one mask, the fingers in the polysilicon layer and singlecrystal silicon layer were etched sequentially. Underneath the polysilicon fingers, the unwanted single-crystal silicon was previously transformed to oxide. This process step makes use of fluorine-based, chlorine-based, and Bosch-process [21] etchers to etch the layers of sacrificial oxide, polysilicon, and single-crystal silicon while the buried oxide of the SOI wafer functions as the etch stop layer. Rapid thermal annealing was performed at the temperature of $1100{ }^{\circ} \mathrm{C}$ for one minute to reduce the interfacial stresses between different materials. Last, the silicon micromachined structures were released using a hydrofluoric acid solution to remove all the sacrificial oxide [see Fig. 6(i)].

The reason for separating the polysilicon etch into two parts where the final etch was used for the formation of the movable fingers of the combdrive is to simplify the formation of the polysilicon structures and to gain the maximum dimensional control of the structures. In addition, the separation of the etches allowed us to avoid design limitations such as having the polysilicon structures be the same dimension as the underlying single-crystal silicon structures.

\section{B. A Membrane Actuated by Asymmetric Combdrives and Parallel-Plate Actuators}

For the device with bottom electrodes underneath the polysilicon membrane (see Fig. 5), a few process steps (see Fig. 7) are required in addition to the process delineated in Section III-A. To fabricate this device, we also began with an SOI wafer that had a $20-\mu \mathrm{m}$-thick silicon layer on top of a 1- $\mu \mathrm{m}$-thick buried oxide. First, we created the bottom electrodes of a parallel-plate actuator out of the top silicon layer of the SOI wafer. Trenches that were etched in the top silicon layer with a DRIE etcher using photoresist as the mask were $2-3 \mu \mathrm{ms}$ wide, $1 \mu \mathrm{m}$ apart, and $17 \mu \mathrm{m}$ deep [see Fig. 7(a)]. The remaining 3- $\mu$ m single-crystal silicon was kept for the two underlying plates (electrodes) parallel to the substrate surface.

The deposition of low-temperature oxide (LTO) $(30 \mathrm{~nm} / \mathrm{min}$ at $400{ }^{\circ} \mathrm{C}$ ) covered the deep trench openings and smoothed the silicon surface for the next photolithographic step [see Fig. 7(b)]. The LTO also served as a hard mask for the next deep etch where the buried oxide of the SOI wafer was the etch stop layer. The 17- $\mu \mathrm{m}$-thick silicon posts on top of the underlying electrodes were protected by the deposited LTO from the deep etch, which was $20 \mu \mathrm{m}$ in depth, and which created the silicon beam structures for the oxide block [see Fig. 7(c)]. To avoid single-crystal silicon residue within the oxide block due to beams in different steps, we overlapped the trench structures. The purpose of the second deep etch is to form the oxide block around the bottom electrodes and under polysilicon structures. A short HP dip was taken to remove the deposited LTO remaining around the beam structures. Thermal oxidation at the temperature of $1050{ }^{\circ} \mathrm{C}$ completely transformed the silicon beams to silicon dioxide [see Fig. 7(d)]. Conformal LPCVD oxide was deposited to fill the open trenches between the fully oxidized beams, and then the resultant bumpy oxide surface was planarized by CMP [see Fig. 7(e)]. The rest of the fabrication procedure after the formation of the oxide block is the same as described in Section III-A.

\section{DeVice Characteristics}

The asymmetric combdrive lowers the natural resonant frequency of the spring-membrane system (see Fig. 5) due to the additional contribution on the moment of inertia. However, for an operation range of few micrometers, replacing a combdrive with a parallel-plate actuator would not generate as large a torque on the spring-membrane system under the same applied voltage and the same angular rotation range [18]. This is because the force per area of a parallel-plate actuator is limited by its operation range (1/3 of separation gap) while the asymmetric combdrive is only restricted by the thickness of the fixed fingers. Therefore, compared to a parallel-plate actuator, the asymmetric combdrive requires a lower operating voltage to actuate the same rotational angle under the condition of devices with the same resonant frequency. 


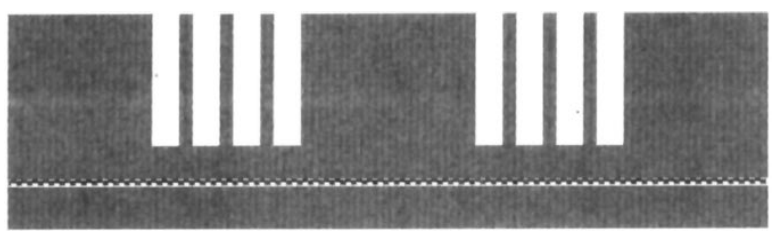

(a)

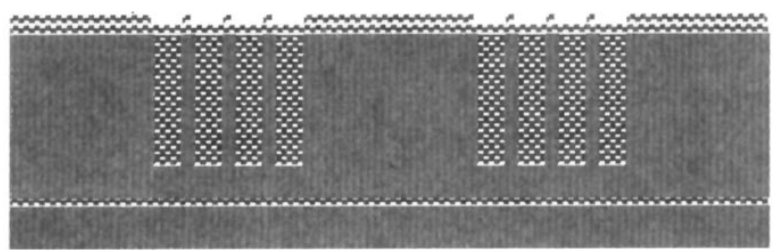

(b)

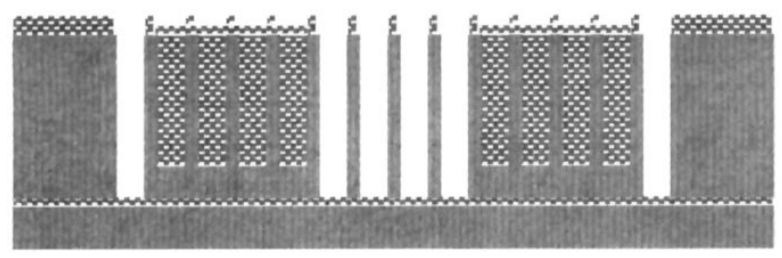

(c)

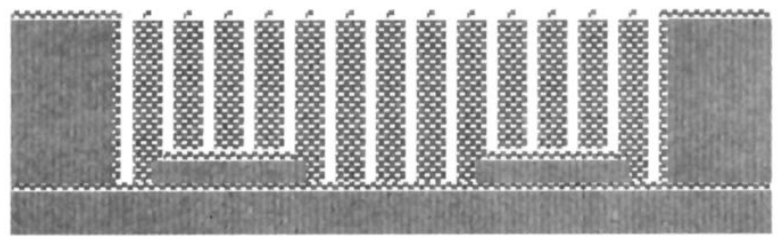

(d)

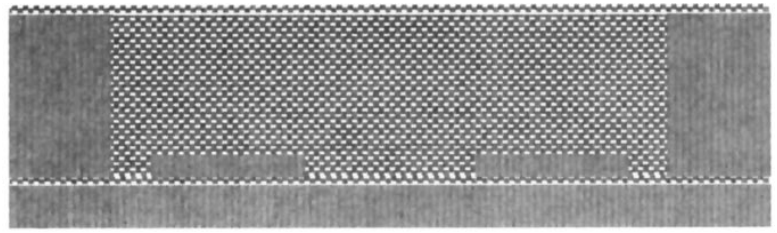

(e)

Fig. 7. Fabrication sequence of the electrodes underneath a membrane. (a) Pattern and etch beam structures $17 \mu \mathrm{m}$ deep using DRIE. (b) Fill trenches with LPCVD oxide. (c) Pattern and etch beam structures through the top silicon layer using DRIE. (d) Thermal oxidation at a temperature of $1050^{\circ} \mathrm{C}$. (e) Refill trenches with LPCVD oxide and then do chemical mechanical polishing.

\section{A. Asymmetric Combdrive Forces and Torques}

The asymmetric combdrive formed by a set of polysilicon movable fingers and bulk silicon fixed fingers is actuated by the fringing capacitance that is due to the different thicknesses and heights of the two combs. The majority of the induced fringing electric field lines in this combdrive point downward toward the substrate rather than in an in-plane direction, which is just the opposite of what occurs in the conventional in-plane interdigitated combdrive. This type of electrostatic combdrive does not have invariant force output with respect to displacement as in an in-plane interdigitated combdrive, though their geometric shapes from the top view look similar.

The output force and torque due to the changing capacitance, which are expressed in (1) and (2), vary with the position of the polysilicon comb fingers.

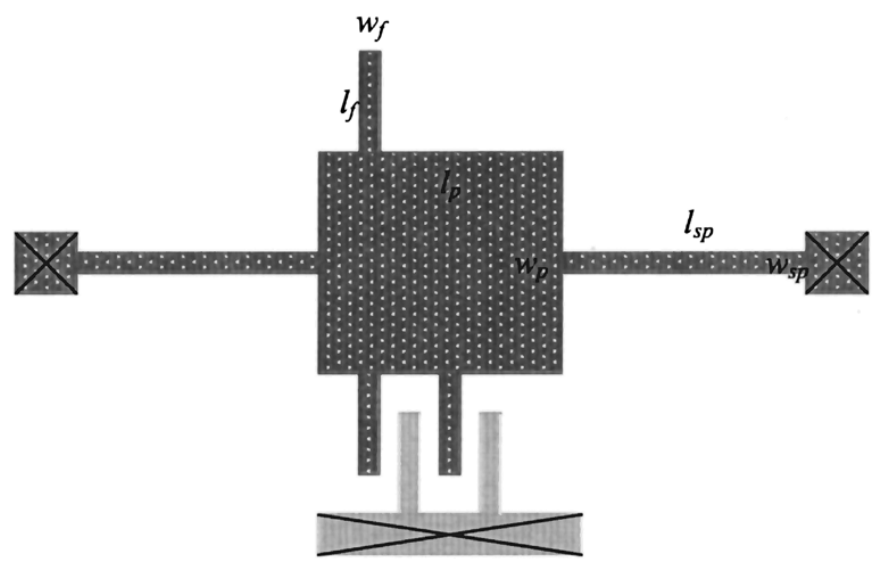

Polysilicon

Bulk silicon

Fig. 8. Overhead schematic of an asymmetric-combdrive actuated membrane.

The force in the into-plane direction is

$$
F_{z}=N_{f} \frac{V^{2}}{2} \frac{\partial C}{\partial z} .
$$

The torque in the angular motion is

$$
T=N_{f} \frac{V^{2}}{2} \frac{\partial C}{\partial \theta} .
$$

\section{B. Influence of the Combdrive on the Motion of a Membrane}

A polysilicon membrane, shown in Fig. 4, is suspended by a pair of rectangular bars and is actuated by asymmetric combdrive. There are dimensional criteria associated with the members of this structure; the structure illustrated in Fig. 8 is designed with these criteria in mind. The following is a discussion of the influence of the additional asymmetric combdrive on the static and dynamic performance of the actuated membrane.

1) Static Response in Torsional Motion: The rotation angle of the torsional plate is defined by the following equation:

$$
\theta=\frac{t}{K_{\phi}}
$$

where the applied torque $T$ is expressed in (2) and the torsional stiffness of springs (polysilicon rectangular beams) $K_{\phi}$ can be described as $K_{\phi}=2 \times\left(G I_{s p} / l_{\mathrm{sp}}\right)$. In this expression, $l_{\text {sp }}$ is the length of each beam and $G$ is the elastic shear modulus of polysilicon, which is related to both Young's modulus $E$ assumed as $170 \mathrm{GPa}$ and Poisson's ratio $v$ equal to 0.22 , according to $G=(E / 2(1+v))$. The moment of inertia of a spring $I_{\mathrm{sp}}$ is expressed as $I_{\mathrm{sp}}=K_{I} R_{\mathrm{sp}} h_{\mathrm{sp}}^{4}$. The parameter $R_{\mathrm{sp}}$ is the ratio of the width of a rectangular spring to its height, $R_{\mathrm{sp}}=\left(w_{\mathrm{sp}} / h_{\mathrm{sp}}\right)$. The geometric factor $K_{I}$ is 
dependent on the spring shape. For a rectangular cross section, $K_{I}$ is given by [22]

$$
K_{I}=5.33 \times \frac{1}{16} \times\left[1-\frac{0.63}{R_{\mathrm{sp}}} \times\left(1-\frac{1}{12 R_{\mathrm{sp}}^{4}}\right)\right] .
$$

According to (3), the system has invariant static response even though the combdrive is attached to it.

2) Dynamic Response in the Torsional Mode: For a damping-free system, the dynamic behavior can be described by the following equation of motion:

$$
I_{p} \ddot{\theta}+K_{\phi} \theta=T
$$

where the moment of inertia $I_{p}$ of a polysilicon membrane and the attached combs is

$$
I_{p}=\rho \times \frac{\left[\left(l_{p}-N_{f} \times w_{f}\right) w_{p}^{3} h_{p}+N_{f} w_{f}\left(w_{p}+2 l_{f}\right)^{3} h_{f}\right]}{12} .
$$

In the expression, the parameters $l_{p}, w_{p}, h_{p}$ denote the length, width, and thickness of the polysilicon membrane, respectively; the parameters $l_{f}, w_{f}, h_{f}, N_{f}$ denote the length, width, thickness, and number of the polysilicon comb fingers, respectively; and $\rho$ is the density of polysilicon, which is 2300 $\mathrm{kg} / \mathrm{m}^{3}$. In this process, all polysilicon structures have the same thickness, i.e., $h_{\mathrm{sp}}=h_{p}=h_{f}$. The natural resonant frequency, which is geometry dependent, is defined as

$$
\begin{aligned}
f_{R, \theta}= & \frac{1}{2 \pi} \sqrt{\frac{K_{\phi}}{I_{P}}} \cong \frac{1}{2 \pi} \\
& \cdot \sqrt{\frac{24 K_{I} G R_{\mathrm{sp}} h_{\mathrm{sp}}^{3}}{\rho l_{\mathrm{sp}}\left[\left(l_{p}-N_{f} \times w_{f}\right) w_{p}^{3}+N_{f} w_{f}\left(w_{p}+2 l_{f}\right)^{3}\right]}} .
\end{aligned}
$$

Compared to the same structure without the asymmetric combdrive, and which is driven by a parallel-plate actuator (i.e., $l_{f}=0$ ), the ratio of the natural resonant frequencies is

$$
\begin{aligned}
\frac{f_{R, \mathrm{PP}}}{f_{R, L C}} & =\sqrt{\frac{I_{p, L C}}{I_{p, P P}}} \\
& =\sqrt{1+\frac{2 N_{f} w_{f}}{l_{p}}\left[3\left(\frac{l_{f}}{w_{p}}\right)+6\left(\frac{l_{f}}{w_{p}}\right)^{2}+4\left(\frac{l_{f}}{w_{p}}\right)^{3}\right] .}
\end{aligned}
$$

From the above result, the asymmetric combdrive lowers the natural resonant frequency of a device due its mass and position. The dimension of the device, shown in Fig. 5, is measured as follows:

$$
\begin{aligned}
h_{\mathrm{sp}} & =h_{p}=h_{f}=1.5 \mu \mathrm{m} \\
l_{p} & =w_{p}=l_{\mathrm{sp}}=200 \mu \mathrm{m} \\
N_{f} & =17 \\
w_{f} & =1.8 \mu \mathrm{m} \\
l_{f} & =70 \mu \mathrm{m} \\
w_{\mathrm{sp}} & =1.9 \mu \mathrm{m} .
\end{aligned}
$$

The theoretical natural resonant frequency of the device is $f_{R, \theta}=4.9 \mathrm{kHz}$, and the ratio $f_{R, \mathrm{PP}} / f_{R, \mathrm{LC}}$ is equal to 1.32 .
The natural resonant frequency of this device was measured to be $4.7 \mathrm{kHz}$ under a small ac signal with a $10-\mathrm{V}$ dc bias. The dc bias reduces the effective torsional rigidity, resulting in a lower resonant frequency [18], [23].

\section{Comparison Between Asymmetric Combdrive and Parallel-Plate Actuator}

To compare our combdrive with the most popular torsional actuator, the parallel-plate actuator, a dually actuated membrane, shown in Fig. 5, was used for the experimental test. The torsional motion actuated by the combdrive can also be accomplished with a parallel-plate actuator consisting of the micromirror and its underlying electrodes. Both experimental and simulation data of the static response to the driving of the parallel-plate actuator and the combdrive with dc bias are shown in Fig. 9. It is found that the fabricated asymmetric combdrive generates a larger rotational angle than the parallelplate actuator under an equal applied voltage. In our first run, the bottom and top electrodes were separated from each other by a vertical gap of $20 \mu \mathrm{m}$, and the gap between the polysilicon and bulk silicon fingers measured $4.2 \mu \mathrm{m}$ from the top view. Under a $15-\mathrm{V}$ dc bias, the measured rotational angles of the membrane when actuated by the combdrive and the parallel-plate actuator were $0.95^{\circ}$ and $0.41^{\circ}$, respectively. The maximum rotation actuated by asymmetric combdrives was measured to be $1.14^{\circ}$ that corresponds to the vertical deflection of $3.6 \mu \mathrm{m}$ at the ends of the movable fingers. In fact, the maximum of the measured rotation is much smaller than the theoretical value of $2.6^{\circ}$ at $36 \mathrm{~V}$ [18]. The reason is that the fabricated combdrives (prototype) reach the instability zone due to a layout mistake. The movable fingers are not wide enough to bear the perturbation of the electric attraction between the combs. To compare the performances of the combdrive and the parallel-plate actuator, the required driving voltage is considered under the conditions of both the same natural resonant frequency and the same rotational angle. By combining (2) and (5), the required driving voltage is expressed as follows:

$$
V=2 \pi f_{R, \theta} \sqrt{2 \theta} \sqrt{\frac{I_{p}}{\left(\frac{\partial C}{\partial \theta}\right)}} .
$$

Thus, according to the experimental data from Fig. 9, the driving voltage required for the parallel-plate actuator is higher than that required for the asymmetric combdrive by $30 \%$, while the resonant frequency and the rotational angle of the device are kept the same. The gap between the polysilicon finger and the bulk silicon finger can be potentially reduced to $2 \mu \mathrm{m}$, and the required driving voltage can be lowered by additional $50 \%$.

\section{Potential Applications And Future Work}

The asymmetric combdrive is not only good for actuation but also has some benefits when used as a torsional resonator. Microelectromechanical resonators do not always have the dynamic responses that one would like. As a matter of fact, some device features such as resonant frequency and spring 


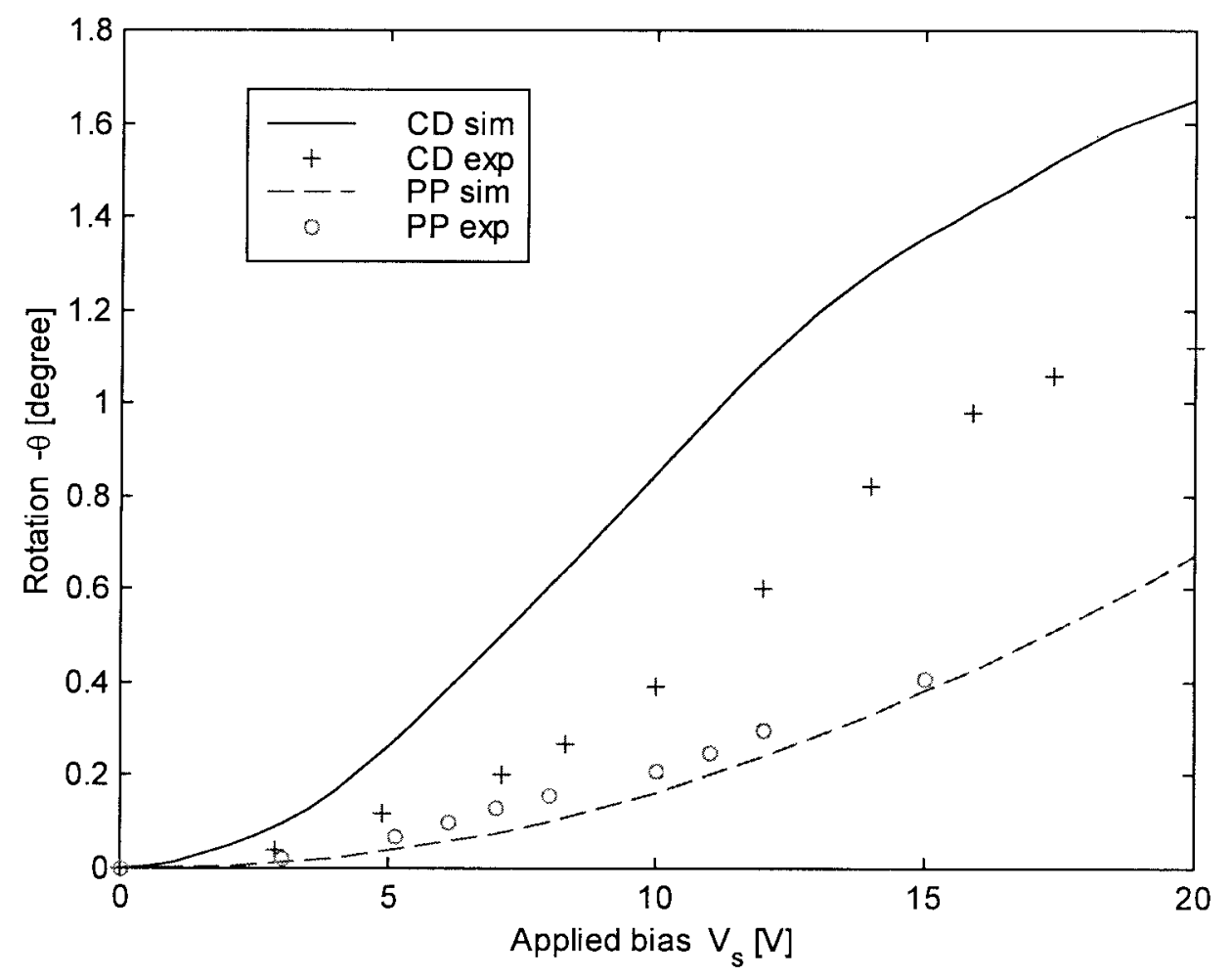

Fig. 9. Experimental and simulation results of dual actuations. CD stands for asymmetric combdrive, PP for parallel-plate actuation, sim for simulation, and exp for experiment.

stiffness may fall out of the desired range due to waferto-wafer or run-to-run variations in fabrication. Used as a torsional resonator, the asymmetric combdrive has the major advantage of being able to tune the resonant frequency by a $\mathrm{dc}$ bias because its differential capacitance varies with respect to the rotation angle. Thus, no additional tuning components or specific frequency-trimming techniques are required. The potential applications of the combdrive include the use as a frequency synchronizer when coupled with a sustaining amplifier and the use as a mechanical filter when several torsional resonators are linked with each other.

A scanning micromirror driven by dual actuators, shown in Fig. 5, configured and used as a system with an internal feedback loop will be tested for the control of angular position or angular rate in the future. The internal feedback loop may shorten the response delay so that a faster control response can be achieved. In addition, the device may reduce the size and the cost of a feedback system. To form a feedback loop, the two underlying plates and the membrane could be used as a differential capacitive sensor, which enhances the amplitude and linearity of the output signals while the asymmetric combdrive actuates the micromirror in torsional motion.

It is straightforward to fabricate more layers of polysilicon structures on top of the SOI wafer. The present process was one polysilicon layer and one single-crystal silicon layer for the formation of movable structures. With two movable polysilicon layers, the integrated process gains more design flexibility. An example would be the fabrication of hinged polysilicon structures [16], [24] directly sitting on singlecrystal silicon movable platforms that in conventional surface micromachining might require four-layer polysilicon surface micromachining. Compared to four-layer polysilicon surface micromachining, our integrated technique not only reduces the process topography but also provides a thick single-crystal silicon layer for large force output, high resonant frequency, or heavy proof mass. Furthermore, the integrated process could eliminate mechanical coupling [24] between different structures that are linked to each other by a hinge. A hinge can transfer the energy forward and backward between translational motion and rotational motion. However, the coupling increases the system instability if two structures connected by a hinge have overlapped modal frequencies. Modal frequency separation can be achieved by having the structures fabricated using either polysilicon surface micromachining or DRIE bulk silicon micromachining because the resonant frequency of a structure is mainly inherent to the micromachining process.

\section{ACKNOWLEDGMENT}

The authors are grateful to Prof. E. Kan for rewarding discussion, the entire Cornell Nanofabrication Facility staff for their support, and Z. Liu, D. Gan, J. Chong, and C. Pu for their assistance in simulation, fabrication, and measurement.

\section{REFERENCES}

[1] K. A. Shaw, Z. L. Zhang, and N. C. MacDonald, "SCREAM I: A single mask, single-crystal silicon process for microelectromechanical structures," in Proc. IEEE Micro Electro Mechanical Systems Workshop (MEMS '93), Fort Lauderdale, FL, 1993, pp. 155-160.

[2] W. H. Juan and S. W. Pang, "Released Si microstructures fabricated by deep etching and shallow diffusion," J. Microelectromech. Syst., vol. 5, no. 1, pp. 19-23, 1996. 
[3] M. Rodgers and J. Sniegowski, "5-level polysilicon surface micromachine technology: Application to complex mechanical systems," in Proc. IEEE Solid State Sensors and Actuators Workshops (Hilton Head'98), June 1998, pp. 144-149.

[4] W. C. Tang, T.-C. H. Nguyen, M. W. Judy, and R. T. Howe, "Electrostatic combdrive of lateral polysilicon resonators," Sens. Actuators, vol A21-23, pp. 328-331, 1990.

[5] T. Akiyama and K. Shono, "Controlled stepwise motion in polysilicon microstructures," J. Microelectromech. Syst., vol. 2, no. 3, pp. 106-110, 1993.

[6] H. Guckel, J. Klein, T. Christenson, K. Skrobis, M. Laudon, and E. Lovell, "Thermo-magnetic metal flexure actuators," in Proc. IEEE Solid State Sensors and Actuators Workshops (Hilton Head'92), June 1992, pp. 73-75.

[7] M. Lutz, W. Golderer, J. Gerstenmeier, J. Marek, B. Maihofer, S. Mahler, H. Munzel, and U. Bischof, "A precision yaw rate sensor in silicon micromachining," in Proc. 9th Int. Conf. Sensors and Actuators (Transducer '97), Chicago, IL, June 1997, pp. 847-850.

[8] K. E. Peterson, "Silicon torsional scanning mirror," IBM J. Res. Develop., vol. 24, no. 5, 1980, pp. 631-637.

[9] L. Hornbeck, "Current status of the digital micromirror device (DMD) for projection television applications," in Proc. IEEE Int. Electron Devices Meeting, Washington, DC, Dec. 1993, pp. 381-384.

[10] D. L. Dickensheet and G. S. Kino, "Silicon-micromachined scanning confocal optical microscope," J. Microelectromech. Syst., vol. 7, no. 1, pp. 38-47, 1998.

[11] T. Gessner, W. Dotzel, D. Billep, R. Hahn, C. Kaufmann, S. Kurth, K. Kehr, C. Steiniger, and U. Wollmann, "Silicon mirror arrays fabricated by using bulk- and surface-micromachining," in Proc. SPIE, San Jose, CA, Feb. 1997, vol. 3008, pp. 256-305

[12] W. Dotzel, T. Gessner, R. Hahn, C. Kaufmann, K. Kehr, S. Kurth, and J Mehner, "Silicon mirrors and micromirror arrays for spatial laser beam modulation," in Proc. 9th Int. Conf. Sensors and Actuators (Transducer '97), Chicago, IL, June 1997, pp. 81-84.

[13] J. Kraenert, C. Deter, T. Gessner, and W. Dotzel, "Laser display technology," in Proc. IEEE Micro Electro Mechanical Systems Workshop (MEMS '93), Heidelberg, Germany, 1998, p. 99.

[14] S. A. Miller, K. L. Turner, and N. C. MacDonald, "Microelectromechanical scanning probe instruments for array architectures," Rev. Sci. Instrum., vol. 68, Nov. 1997, pp. 4155-4162.

[15] W. C. Tang, M. G. Lim, and R. T. Howe, "Electrostatic comb drive levitation and control method," J. Microelectromech. Syst., vol. 1, no. 4, pp. 170-178, 1992.

[16] M.-H. Kiang, O. Solgaard, K. Y. Lay, and R. S. Muller, "Electrostatic combdrive-actuated micromirror for laser-beam scanning and positioning," J. Microelectromech. Syst., vol. 7, no. 1, pp. 27-37, 1998

[17] N. C. Tien, M.-H. Kiang, M. J. Daneman, O. Solgaard, K. Y. Lau, and R. S. Muller, "Actuation of polysilicon surface-micromachined mirror," in Proc. SPIE 1996 Int. Symp. Lasers and Optoelectronics, San Jose, CA, vol. 2687, 1996, pp. 53-59.

[18] J.-L. A. Yeh, N. C. Tien, and C.-Y. Hui, submitted for publication.

[19] H. Toshiyoshi and H. Fujita, "Electrostatic micro torsion mirrors for an optical switch matrix," J. Microelectromech. Syst., vol. 5, no. 4, p. 231, 1996.

[20] H. B. Erzgraber, T. Grabolla, H. H. Richter, P. Schley, and A. Wolff, "A novel buried oxide isolation for monolithic RF inductors on silicon," in Proc. Int. Electron Devices Meeting (IEDM '98), Dec. 1998, pp. 535-539.

[21] F. Laermer and A. Schilp, "Method of anisotropically etching silicon," U.S. Patent 5501 893, Aug. 5, 1994.

[22] G. M. Gere and S. P. Timoshenko, Mechanics of Materials, 3rd ed. Boston, MA: PWS-KENT, 1990
[23] K. B. Lee and Y.-H. Cho, "A triangular electrostatic comb array for micromechanical resonant frequency tuning," Sens. Actuators, vol. 70, pp. $112-117,1998$

[24] R. S. Muller and K. Y. Lau, "Surface-micromachined microoptical elements and systems," Proc. IEEE, vol. 86, pp. 1705-1720, Aug. 1998

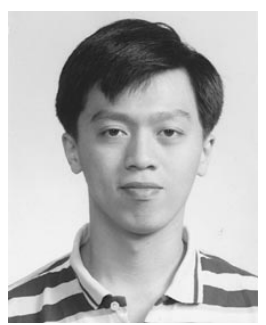

Jer-Liang Andrew Yeh (S'97) received the B.S. degree in mechanical engineering from the National Taiwan University, Taiwan, R.O.C., in 1992 and the M. Eng. degree in mechanical engineering and in electrical engineering from Cornell University, Ithaca, NY, in 1996 and 1997, respectively, where he is currently pursing the Ph.D. degree in electrical engineering.

His research interests include design and fabrication of MEMS systems, micromechanics issues in micromachined structures, and MEMS elements for

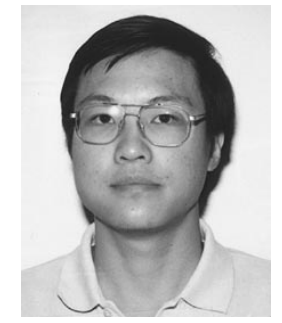

Hongrui Jiang (S'98) was born in Xi' an, China, in 1973. He received the B.S. degree in physics from Peking University, China, in 1995. He is currently pursuing the Ph.D. degree in electrical engineering at Cornell University, Ithaca, NY.

His research interest is mainly in applications of MEMS in RF circuits.

Norman C. Tien (S'87-M'89) received the B.S. degree from the University of California, Berkeley, the M.S. degree from the University of Illinois, UrbanaChampaign, and the Ph.D. degree from the University of California, San Diego in 1993.

$\mathrm{He}$ is an Assistant Professor in the School of Electrical Engineering at Cornell University, Ithaca, NY, where his research interest is in the development of silicon microelectromechanical systems (MEMS), including the design and fabrication of microactuators, microsensors, micromechanical structures, and systems. From 1993 to 1996, he was a Lecturer in the Department of Electrical Engineering and Computer Science at the University of California, Berkeley, and a Postdoctoral Research Engineer associated with the Berkeley Sensor \& Actuator Center. Between 1984 and 1986, he was a Silicon Process Development Engineer with Polaroid Corp., Cambridge, MA. 